\title{
Vague neck pain following a gastrointestinal procedure: a rare case presentation of vertebral osteomyelitis and cervical epidural abscess
}

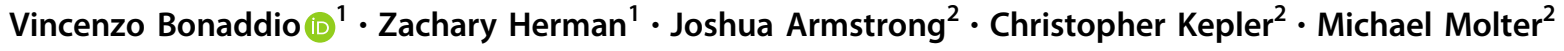

Received: 16 April 2020 / Revised: 30 April 2020 / Accepted: 1 May 2020

(C) International Spinal Cord Society 2020

\begin{abstract}
Introduction Vertebral osteomyelitis (VO), spondylodiscitis, and spinal epidural abscesses (SEA) are infectious and inflammatory processes impacting the spine that cause major morbidity and mortality. They require prolonged hospital stays with expensive treatment regimens. Along with acute management, studies have provided evidence highlighting poor longterm outcomes. VO accounts for $\sim 2 \%$ of all osteomyelitis. Recent data have illustrated an increase in incidence to 5.4 per 100,000 person years. The majority of patients that present with SEA and VO typically have some combination of back pain, fevers, and neurological deficits.

Case presentation A 55-year-old woman with known history of hypertension and hyperlipidemia, status-post endoscopic repair of a Zenker's Diverticulum 3 weeks prior, presented to our outpatient clinic with a 2-week history of axial cervical spine pain as well as left sided scapular and deltoid pain. Further questioning and exam revealed no neurologic deficits or fever. As pain persisted and she did not respond to treatment, further imaging was performed. She was found to have cervical discitis, osteomyelitis, and a cervical epidural abscess.

Discussion Patients presenting with VO/SEA typically have spine pain with some other associated symptoms of spinal cord compression or fevers, making this a rare presentation. The urgency for discovery because of the need for emergent operative intervention is evident by the documented complications associated with a delay in diagnosis. This case report emphasizes the importance of always keeping VO/SEA on the differential for cervical spine pain even with lack of associated symptoms, in order to optimize patient care.
\end{abstract}

\section{Introduction}

Vertebral osteomyelitis (VO), spondylodiscitis, and spinal epidural abscesses (SEA) are infectious and inflammatory processes impacting the spine that cause major morbidity and mortality. They commonly require prolonged hospital stays with expensive treatment regimens including extensive imaging, antibiotics, and oftentimes surgical intervention.

These authors contributed equally: Vincenzo Bonaddio,

Zachary Herman

Zachary Herman

Zjh001@jefferson.edu

1 Sidney Kimmel Medical College at Thomas Jefferson University, Philadelphia, PA, USA

2 The Rothman Orthopaedic Institute at Thomas Jefferson University, Philadelphia, PA, USA
Along with acute management, multiple studies have provided evidence highlighting the poor long-term outcomes secondary to severe disability from back pain, impaired mobility, motor weakness/dysfunction, neurogenic bladder, and paralysis [1-3].

In the literature, $\mathrm{VO}$ accounts for $2 \%$ of all osteomyelitis. The incidence was previously estimated to be 2 cases per 100,000 person years, but recent data have illustrated an increase in incidence. One study in the United States illustrated 5.4 cases per 100,000 in 2013 [4]. Hutchinson et al. evaluated patients over the age of 65 and found an incidence of 9.8 cases per 100,000 person years [5]. It is hypothesized that this increase in incidence is attributed to the aging population, increase in intravascular devices and instrumentation causing bacteremia, increase in patients on immunosuppressive therapy, and the improved diagnostic availability of MRI [4].

Regardless of the cause, the increasing incidence of VO poses a great healthcare burden on both the economy and the welfare of our patients. 


\section{Case presentation}

A 55-year-old woman with a known history of hypertension and hyperlipidemia presented to our outpatient clinic with a 2-week history of axial cervical spine pain as well as left sided scapular and deltoid pain. She recently underwent an endoscopic Zenker's Diverticulum repair 3 weeks before presentation. Her continual cervical spine pain required both urgent care and emergency department visits in the preceding 2 weeks, where she had a normal clinical evaluation. She was treated with an oral steroid course and told to follow up in our clinic.

On initial presentation to our clinic, she complained of $7-8 / 10$ cervical spine and left sided arm pain. The pain did not radiate below the level of the elbow. She denied any previous history of these symptoms and associated it with abnormal positioning for a moderate amount of time during her Zenker's Diverticulum repair. There were no other precipitating events. The pain was exacerbated by movements. In addition to oral steroids, she had tried hydrocodone and cyclobenzaprine without relief of her symptoms. On review of systems, she reported recent headache, sleep disturbance, dysphagia, nausea, and aforementioned musculoskeletal pain. She denied numbness or tingling in her extremities, weight change, or bowel/bladder incontinence.

On physical examination, she had decreased cervical range of motion both in right and left rotation secondary to cervical spasms on both sides. She had a positive Spurling's test on the left, which was negative on the right. The rest of her exam was within normal limits. Her upper and lower extremity pulses were intact. She had no spinous process tenderness. Cervical spine flexion and extension were normal, and her scapular motion was normal. She had full range of motion in her upper and lower extremities with 5/ 5 strength. Her reflexes were $2^{+} / 4$. Her tone was normal for age; neither Babinski's nor Hoffmann's reflex was documented. Sensation to light touch was intact in the upper extremities.

An X-ray of her cervical spine was obtained on initial presentation, and it demonstrated straightening of her cervical lordosis with disc space narrowing at $\mathrm{C} 4-\mathrm{C} 5$. The rest of the radiograph was unremarkable.

She was diagnosed with an exacerbation of cervical degenerative disc disease and treated with physical therapy, etodolac, and a trial of gabapentin. Her plan was to follow up in 6 weeks but call back if symptoms worsened or progressed.

Six days after initial evaluation in our outpatient clinic, she called our office with severe, worsening pain. An outpatient MRI was ordered. The next evening, the radiologist that read her report urgently called our office with her results: "C6-C7 discitis with associated osteomyelitis

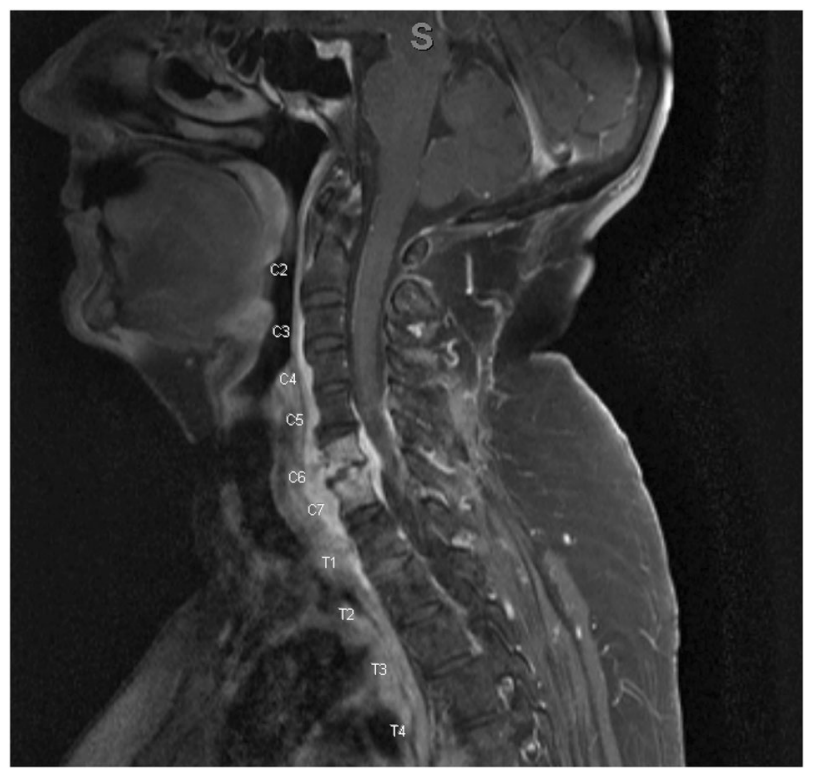

Fig. 1 MRI results in the sagittal plane. Findings were read as: "C6-C7 discitis with associated osteomyelitis with significant anterior prevertebral soft tissue edema and marked phlegmon change extending from C5-T1 along the left anterior aspect of the vertebral bodies. It extends to the left lateral aspect of the vertebral bodies of C6-C7. A $9 \mathrm{~mm}$ abscess is seen anterior to the C7 vertebral body".

with significant anterior prevertebral soft tissue edema and marked phlegmon change extending from C5-T1 along the left anterior aspect of the vertebral bodies; it extends to the left lateral aspect of the vertebral bodies of $\mathrm{C} 6-\mathrm{C} 7$; a $9 \mathrm{~mm}$ abscess is seen anterior to the $\mathrm{C} 7$ vertebral body" (Fig. 1). The patient was called, and she confirmed continued severe neck pain with progressive radiation into the shoulder. She had pain-limited weakness but no new onset upper extremity weakness. She denied bowel or bladder incontinence, worsening balance deficits, fevers, or chills. She was instructed to immediately go to the emergency room.

In the emergency department, she continued to complain of worsening left shoulder and neck pain. Her physical exam was unchanged from the previous encounter described above. She was afebrile with a normal white blood cell count. Otolaryngology, Infectious Disease, and Orthopedics were consulted. Per otolaryngology, she denied any fevers or worsening of her preoperative dysphagia. A noncontract CT scan was performed demonstrating osteomyelitis of C6-7 without obvious esophageal injury (Fig. 2). She was started on antibiotics and prepared for the operating room. An endoscopy was performed and no gross injury to the esophagus was found. She then underwent an anterior cervical discectomy with interbody fusion of C6-7 using leftsided iliac crest autograft bone and posterior cervical instrumented fusion of C6-7 without complication. Wound cultures were obtained in the operating room. 


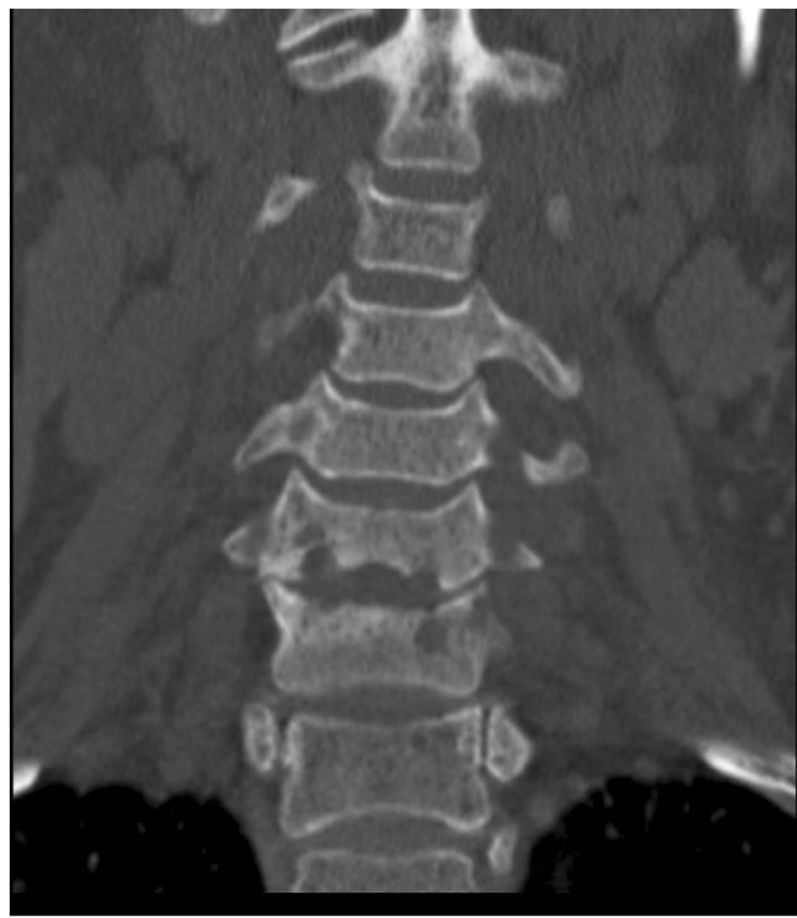

Fig. 2 CT image results. The patient's CT image consistent with osteomyelitis, particularly "irregularity and erosion of the opposing endplates of C6 and C7 vertebral bodies".

The following day, our patient underwent a barium swallow study that showed significant prevertebral soft tissue edema and a small focus of air within the edematous soft tissues along the posterior esophagus at the level of the C6 vertebral body. There was leakage of contrast material into the air-filled space within the edematous prevertebral soft tissues. She was then made nil per os, a Dobhoff feeding tube was placed, and she was continued on IV antibiotics. One anaerobic culture demonstrated moderate growth of Neisseria. A peripherally inserted central catheter was placed and repeat barium swallow was performed demonstrating no leakage of fluid. She was discharged 10 days after initial presentation to the ED.

Postoperatively, she followed up with Otolaryngology and had improving dysphagia. Infectious Disease followed her with a 12-month course of antibiotics for gram-negative SEA. She also followed up with Orthopedics for 1 year and had complete resolution of her preoperative neck and left sided shoulder pain. She made a full recovery and has not had any complications since the surgery.

\section{Discussion}

Infectious processes of the vertebral bodies and intervertebral discs, or spondylodiscitis, are common inflammatory disorders that account for $2 \%$ of all osteomyelitis.
They may occur spontaneously or as result of spinal surgery, mainly surgery for degenerative processes affecting the discs. Patients with diabetes and systemic or lung infections, those undergoing dialysis or transplants, and those whose immune systems are suppressed are more commonly affected. Spondylodiscitis may have an acute or chronic course. The often mild clinical symptomatology and the long latent stage between the onset of symptoms and the appearance of the radiologic changes (from 2 weeks to 8 weeks) may preclude an early diagnosis [6].

Rapid diagnosis may be hindered by lack of typical signs and symptomatology on presentation. In 2002, McHenry et al. reported on presenting signs and symptoms of vertebral osteomyelitis as well as outcomes. Sites of VO were divided based on level, and patients in the cervical VO group were most likely to present with motor dysfunction (44\%) [7]. In addition, Hopkinson et al. reported on presentations of 22 cases of septic discitis in 2001. In $91 \%$ of patients, back pain was the presenting symptom with neurological signs evident in $45 \%$. Fever $>37.5^{\circ} \mathrm{C}$ was present in $68 \%$ of patients, and a marked elevation of erythrocyte sedimentation rate (ESR) in $91 \%$ [8].

In 2000, Reihsaus et al. reported on common signs and symptomatology of patients presenting with SEA. Of the 915 patients, $71 \%$ had back pain as the initial symptom and $66 \%$ had fever. Other common presenting symptoms included muscle weakness and sphincter incontinence as well as sensory deficits. Paralysis was seen on presentation in $34 \%$ of the patients. The average leukocyte count was $15,700 / \mu 1$, and the average ESR was $77 \mathrm{~mm}$ in the first hour [9]. In 2016, Cornett et al. reported that $>85 \%$ of patients with pyogenic VO present with neck or back pain of insidious onset, and $\sim 30 \%$ have neurologic deficits. They found that $35 \%$ to $60 \%$ presented with nonspecific findings of fevers, weight loss, nausea/vomiting, anorexia, lethargy, and confusion. In patients with concomitant spinal epidural abscess, as identified in our patient, they found that most patients present with back pain or motor, sensory, or reflex deficits. Less than $10 \%$ present with incontinence, paraplegia, or quadriplegia. $75-95 \%$ will present with focal tenderness at the specific level of the abscess [1].

Furthermore, delay in diagnosing VO and SEA can have detrimental effects on outcomes because of the resulting delay in surgical intervention. In fact, only rapid and correct management may prevent irreversible neurologic deficits and spinal deformities. Though this topic has been debated, studies have shown the advantage of early diagnosis and intervention in SEA patients. The 2002 study by McHenry et al. found time to diagnosis to be an independent risk factor for adverse outcomes (defined as death, sustained pain, motor weakness or paralysis, impaired mobility, or neurogenic bladder). The median interval between the onset of symptoms and diagnosis was significantly longer for 
patients with adverse outcomes (2.1 months) than it was for those who recovered (1.4 months; $p=0.025$ ) [7].

In 2004, Davis et al. reported on the delay to diagnose SEA and the associated detrimental outcomes. Inpatients with a discharge diagnosis of SEA and a related visit before the admission were identified over a 10-year time period. A delayed diagnosis was defined as either: (1) multiple visits before diagnosis, or (2) admission without a diagnosis of SEA and $>24 \mathrm{~h}$ before a definitive study was obtained. A pool of patients presenting with a chief complaint of spine pain was generated. A total of 63 SEA patients were handmatched to 126 controls with spine pain. Diagnostic delays were present in $75 \%$ of SEA patients. Of the SEA patients, residual motor weakness was present in $45 \%$ of patients on discharge versus only $13 \%$ of patients without diagnostic delays $(p<0.05)[2]$.

In addition, a retrospective review in December 2018 by Segreto et al. evaluated the effects on delayed surgery in patients with VO. They assessed patients who underwent VO surgery from 1998 to 2013 and stratified them based on the number of days the procedure was delayed. Regression analysis showed that delaying surgical intervention was the strongest independent indicator of mortality, neurologic deficit, or any complication $(p<0.001)$ [10]. While this study is limited because time to diagnosis was unclear, it can be assumed that an earlier diagnosis would expedite surgical intervention if necessary.

As evidenced in the studies above, the majority of patients that present with SEA and VO typically have some combination of back pain, fevers, and neurological deficits such as muscular weakness or sensory deficits. Our patient had a unique presentation in relation to the reported literature given the lack of symptoms as well as the cause of her VO/SEA after gastrointestinal manipulation. She presented with axial cervical spine and left shoulder pain that mimicked degenerative disc disease in the cervical spine. She remained afebrile throughout, her strength on examination was $5 / 5$ bilaterally in all extremities, and her sensation was intact. She also had cervical muscular spasms and radiographic imaging showing loss of her cervical lordosis: signs much more consistent with degenerative disc disease. This confounding and lack of clinical evidence led to a delay in her diagnosis, which could have had detrimental effects on her long-term outcomes, as evidenced above with increased mortality, neurologic deficit, or any complication.

This case report shows a rare presentation of VO/SEA that should raise awareness of a rather asymptomatic presentation and should act as a reminder to always consider this life-threatening condition on differential diagnosis for axial spine pain, especially shortly after procedures.

\section{Compliance with ethical standards}

Conflict of interest The authors declare that they have no conflict of interest.

Publisher's note Springer Nature remains neutral with regard to jurisdictional claims in published maps and institutional affiliations.

\section{References}

1. Cornett C, Vincent S, Crow J, Hewlett A. Bacterial spine infections in adults. J Am Acad Orthop Surg. 2016;24:11-18.

2. Davis D, Wold R, Patel R, Tran A, Tokhi R, Chan T, et al. The clinical presentation and impact of diagnostic delays on emergency department patients with spinal epidural abscess. J Emerg Med. 2004;26:285-91.

3. Pozo J, Soto M, Solera J. Vertebral osteomyelitis: long-term disability assessment and prognostic factors. J Infect. 2007;54:129-34.

4. Issa K, Diebo B, Faloon M, Naziri Q, Pourtaheri S, Paulino C, et al. The epidemiology of vertebral osteomyelitis in the United States from 1998 to 2013. Clin Spine Surg. 2018;31:102-8.

5. Hutchinson C, Hanger C, Wilkinson T, Sainsbury R, Pithie A. Spontaneous spinal infections in older people. Intern Med $\mathrm{J}$. 2009;39:845-8.

6. Maiuri F, Laconetta G, Gallicchio B, Manto A, Briganti F. Spondylodiscitis. Spine. 1997;22:1741-6.

7. McHenry M, Easley K, Locker G. Vertebral osteomyelitis: Longterm outcome for 253 patients from 7 Cleveland-area hospitals. Infect Dis Clin Pract. 2002;11:169-70.

8. Hopkinson N, Stevenson J, Benjamin S. A case ascertainment study of septic discitis: clinical, microbiological and radiological features. QJM. 2001;94:465-70.

9. Reihsaus E, Waldbaur H, Seeling W. Spinal epidural abscess: a meta-analysis of 915 patients. Neurosurg Rev. 2001;24:175-204.

10. Segreto F, Beyer G, Grieco P, Horn S, Bortz C, Jalai C, et al. Vertebral osteomyelitis: a comparison of associated outcomes in early versus delayed surgical treatment. Int J Spine Surg. 2018;12:703-12. 